\title{
Application of Multiacoustic Data in Feature Extraction of Anemometer
}

\author{
Dawei Chen and Xu Guo ii \\ School of Musice Dance, Qiqihar University, Heilongjiang, Qiqihar 161000, China \\ Correspondence should be addressed to Xu Guo; wanghui10@post.eurasia.edu
}

Received 1 June 2021; Revised 27 June 2021; Accepted 17 July 2021; Published 24 July 2021

Academic Editor: Muhammad Javaid

Copyright (c) 2021 Dawei Chen and Xu Guo. This is an open access article distributed under the Creative Commons Attribution License, which permits unrestricted use, distribution, and reproduction in any medium, provided the original work is properly cited.

\begin{abstract}
The acoustic characteristics of wind instruments are a major feature in the field of vocal music. This paper studies the application effect of wind power instrument feature extraction based on multiacoustic data. Combined with the acoustic data training model, the classification algorithm based on deep trust network is used to process multiple acoustic data. Using multiple acoustic data for feature extraction, the recognition and matching between multiple acoustic data and wind measuring instrument are realized. The experiment not only evaluates the error of the network classification algorithm but also describes the evaluation function of the deep belief network classification algorithm in the system. The traditional SNR evaluation method is used to improve the deficiency of evaluation function. Through the deep belief network classification algorithm for self-learning, the instrument recognition method with strong applicability is established. Finally, the effectiveness of multiacoustic data in wind power instrument feature extraction is verified.
\end{abstract}

\section{Introduction}

In the traditional instrument recognition methods, the recognition and cognition of instruments are generally realized through the angle analysis of the unique characteristics of instruments and in-depth auditory or visual capture [1]. The core content of instrument recognition is to evaluate the accuracy and efficiency of recognition, which is of great value to promote the intelligent development of a variety of acoustic data and instrument combination [2]. In recent years, many mathematicians mainly focus on musical instrument timbre optimization, musical instrument structure improvement, and so on, rarely through acoustic data to study the intelligent instrument recognition system [3]. In China, the research on article feature recognition has a history of decades, involving a lot of content [4]. From the point of view of the evaluation object, it includes conventional cat face recognition, human body recognition, and instrument recognition [5]. At present, the recognition of many acoustic data and wind measuring instruments at home and abroad has the problems of low accuracy and low recognition efficiency. Therefore, many experts and scholars have studied the intelligent and efficient identification methods of multiple acoustic data and wind instruments [6]. However, in the previous research on multiple acoustic data and instrument recognition systems abroad, the methods used are not perfect and there are many fuzziness. Most papers use the deep confidence network classification algorithm to evaluate the effect of singing performance [7].

Ernoult A and other scholars found that the current music evaluation model often adopts the characteristics of fixed frame input and proposed a specific data clustering analysis method to collect audio input information in real time [8]. In order to improve the scoring efficiency of multiple acoustic data, Wang et al. proposed an innovation evaluation method based on the neural network algorithm and related theories [9]. Pan et al. have studied the influence of breathing on the sound quality of musical instruments 
and established an evaluation system. The innovative scoring system has the advantages of high stability and objective evaluation, which is suitable for scoring and analyzing the singing quality of different groups [10]. On the basis of existing research methods, Wang et al. have carried out comparative analysis on different types of wind instruments, combined acoustic data with timbre characteristics of instruments, carried out innovative application and quantitative comparative analysis on different dimensions, and completed the optimization analysis of different sizes of instruments through the design of relevant experiments [11]. Perera and other scholars put forward a new "end-to-end" multiacoustic data ladder scoring system through the research and analysis of singers' singing and timbre differences in different songs and verified the effectiveness of the system in the objective evaluation of singing process through practice [12]. On the basis of music analysis, scholars such as Martin et al. proposed that attention should be paid to the development and construction of the multiple acoustic data analysis system based on restrictive factors so as to realize the management and attention of data information in the process of singing scoring [13]. Lagoida and other scholars proposed a multivariate acoustic data evaluation model based on the neural network algorithm on the basis of the existing information input mode of multivariate acoustic data evaluation model. The input data signal was collected into the evaluation model by the normalization method, and the information normalization processing of data signal was realized by the neural network algorithm [14]. According to the performance difference between multiple acoustic data, Rollinsand other scholars proposed targeted improvement strategies for the recognition of different multiple acoustic data [15]. Vidhyavathi and other scholars found that most colleges and universities still use the traditional music evaluation concept, ignoring the use of intelligent information technology, so they proposed a method of instrument quality evaluation based on intelligent information processing [16].

At present, most of the research studies on instrument feature recognition do not involve the network classification algorithm based on depth confidence and multiacoustic data analysis. Although China has done a lot of basic research on several acoustic data and anemometers, the research on feature recognition of acoustic data and anemometers is relatively less. In this paper, a feature recognition system of the anemometer based on the belief network classification algorithm and multiacoustic data is studied. The first part introduces the research status of multiacoustic data and instrument recognition at home and abroad. In the second part, the point product calculation model of singing data vector and the evaluation index system affecting the recognition quality are established by using the time-domain deep confidence network classification algorithm. In the third part, the experimental verification of the system is carried out, and the experimental results and errors are analyzed.

\section{Construction of Multiple Acoustic Data Analysis Model Based on Deep Belief Network Classification Algorithm}

\subsection{Basic Idea of Deep Belief Network Classification Algorithm.} The characteristics of the instrument refer to the distinctive features different from other instruments and have unique identification. It is usually the representative uniqueness of the instrument in the whole. The musical instrument features are similar to those in other arts. The relatively stable, internal, and profound performance can reflect the external imprint of the internal characteristics of the times, nationalities, or musicians' personal ideas, aesthetic ideals, and spiritual temperament. The formation of the culture of musical instruments is that the times, nationalities, or musicians have surpassed the immature stage in understanding and realizing music, and they have been free from the shackles of various modes, thus tending to or reaching the mature mark. Among many methods of identifying and analyzing multiacoustic data, the deep confidence network classification algorithm is one of the most important methods in recent years. This quantitative method is combined with the mathematical method and information method, and it is used in solving information system problems in the early stage. In recent years, the deep confidence network classification algorithm has been widely used in many industries, which mainly helps enterprises and researchers to solve the problem of specific target recognition, and optimization methods in time and frequency domain are emerging constantly. Figure 1 shows a logic method commonly used in data analysis. These methods are difficult to analyze comprehensively. However, the gray system is different from fuzzy mathematics. This method pays more attention to the objects with clear extension and unclear extension, and the cross section is larger, and permeability is stronger.

Deep belief network algorithms can not only be used to identify features and classify data but also can be used to generate data. The DBN algorithm is a very practical learning algorithm with a wide range of applications and strong scalability. It can be used in machine learning handwriting recognition, speech recognition, image processing, and other fields.

The modeling of the deep belief network classification algorithm is to determine the mathematical relationship between many factors in the gray system, then extract and classify the differential features of multiple acoustic data and wind instruments, and then match and analyze the information stored in the known database. In recent years, with the deepening of the research on the deep belief network classification algorithm, its application in various fields is gradually increasing, so it begins to combine with other algorithms. For example, in the application field of food production, it has been able to provide data support for production development. In the medical field, the application of the deep confidence network classification algorithm 


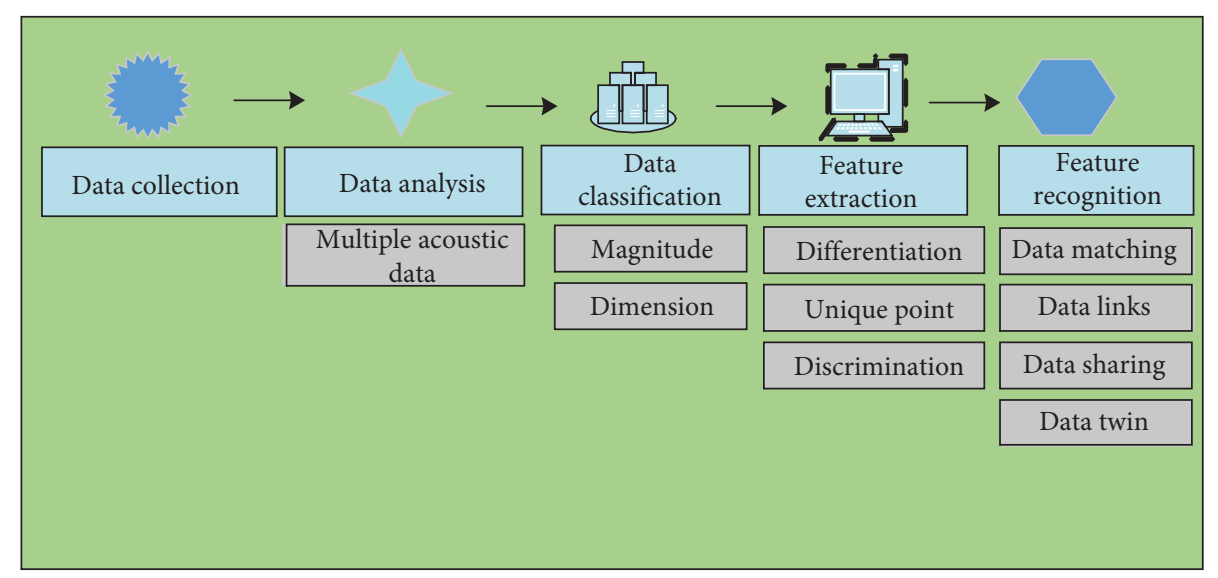

FIgURE 1: A logic method commonly used in data analysis.

can promote the development of medical objectification [17]. In terms of theoretical research, the English version of international academic journals founded by the United States published many studies on this theory, especially in the application of the social system [18]. Imaging, kinematics, acoustic signal processing, and other systems contain various factors, which can be analyzed by using the theory of the deep belief network classification algorithm [19].

\subsection{The Construction of Multiacoustic Data Analysis Model} Based on Deep Confidence Network. In the process of building the multiacoustic data analysis model, this study first selects three parameters related to the multiacoustic data and wind instrument features through the deep confidence network classification algorithm based on adaptive learning factor and proposes a multiacoustic data and instrument recognition management system based on dictionary learning and neighborhood regression [20]. On this basis, the system can not only realize the daily recording, analysis, and storage of different wind instruments and multiple acoustic data but also make full use of the timbre difference between each wind instrument and realize the closed-loop evaluation of the instrument in the performance process through data comparative analysis [21]. On the other hand, the system also uses SNR difference factor to quantitatively describe the data matching degree between the comparison column and the reference column and the difference amount of standard data and uses quantitative indicators to complete the priority of musical instrument classification standards, which can efficiently analyze and extract the factors that affect the quality of musical instrument recognition [22]. The process of multiple acoustic data analysis is shown in Figure 2.

The multiple acoustic data analysis model based on deep belief network clearly defines the hierarchical framework and hierarchical subordination between the whole multiple acoustic data and the instrument recognition system through the research of the zero crossing rate of the signal to be measured, the signal waveform conversion, the dictionary matrix, and the dot product calculation of glottic excitation signal vector. This paper

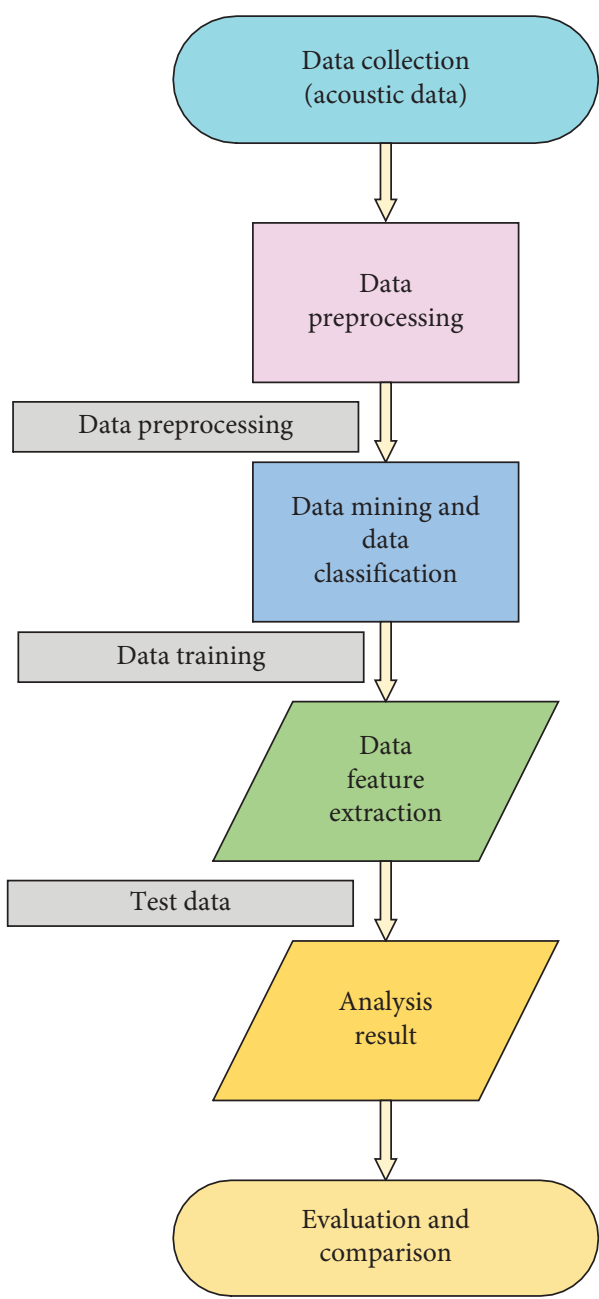

FIGURE 2: The process of analyzing multiple acoustic data.

evaluates the accuracy of the system from many aspects and provides reference samples for the establishment of intelligent multiacoustic data and instrument recognition system [23]. Then, the deep confidence network classification algorithm is used for feature analysis and integrity verification of the classification results. Based on this, the construction process of this model is as follows. 
First of all, the correlation degree is measured according to the relationship or similarity between factors. The basic idea is to sort according to the correlation degree between multiple acoustic data. In the application of this model, the original music data matrix is initialized, and then the reference data column is formulated. Here, the formula of multiple acoustic data eigenvalue, wind instrument eigenvalue, and satisfaction $s$ in accordance with three overview intervals is shown in formulas (1)-(3). The demand condition of one side is represented by $d$, the actual value of the corresponding individual on the other side is $P$, the average value of all individuals on the other side is $p_{\text {mean }}$, the maximum value of the largest individual is $p_{\max }$, the minimum value of the smallest individual is $p_{\min }$, the willingness interval of one side is $d$, the acceptance standard value of one side is $c$, the expected value is $p_{\text {exp }}$, and the satisfaction is $s$.

$$
s=\left(\frac{p_{\max }-p_{\text {mean }}}{p_{\max }-p_{\min }}\right)^{\left(p_{\text {mean }} / c\right)} .
$$

Formula (1) in multiple acoustic data analysis shows that when the satisfaction calculation condition is $p \geq c$, the satisfaction is zero.

$$
s^{\prime}=\left(\frac{p_{\max }-p_{\text {mean }}+\varepsilon}{p_{\max }-p_{\min }+\varepsilon}\right)^{\left(p_{\text {mean }} / c\right)\left(p / p_{\exp }\right)} .
$$

According to the wind instrument recognition formula (2), when the satisfaction calculation condition is $p \leq c$, the satisfaction is zero.

$$
s^{\prime \prime}=\left(\frac{p_{r}-p_{n}+\varepsilon}{p_{R L \max }-p_{\min }+\varepsilon}\right) .
$$

In compound formula (3), the right end point of one individual in $d$ area is represented by $p_{r}$. The left endpoint of the corresponding individual on the other side is represented by $p_{n} \cdot p_{R L \max }$ represents the maximum allowable error of one individual under the same conditions. The larger the value is, the better the degree of satisfaction $s$ is.

\section{Wind Instrument Feature Extraction and Evaluation System Based on Multiple Acoustic Data Analysis Model}

3.1. The Construction of the Evaluation System for the Feature Extraction of the Instrument Based on the Comprehensive Evaluation Method. The comprehensive evaluation method is also called multi-index comprehensive evaluation method. It refers to the method of using more systematic and standardized methods to evaluate multiple indexes and units at the same time. It is not just a method but a method system which refers to a series of effective methods for the synthesis of multiple indicators. Comprehensive evaluation is a multicriteria decision analysis method combining qualitative and quantitative analysis. It is the most widely used decision analysis method for all kinds of problems. It decomposes the relevant factors of decision problems into multiple levels and then carries out qualitative and quantitative analysis. In this model, the analysis process of multiple acoustic data can be analyzed and evaluated from three aspects: dimension, amplitude, and frequency. The process is shown in Figure 3.

In the comprehensive evaluation method used in the wind instrument feature extraction model, the problems are identified first, the system objectives are determined, and the scope and policies involved in the decision-making problems are analyzed. Firstly, the analytic hierarchy process (AHP) system is established, and each index is determined according to different angles, and then the system is divided into different levels. For the convenience of calculation, the block diagram is often used to explain. If there are many factors involved, the hierarchy can be further decomposed. This level division reflects the subordination of each level, but the importance of each index is not the same. At present, the main comprehensive evaluation method is 9-scale method. The evaluation matrix is established based on PSNR characteristics, and then useful information is extracted from the matrix. By constructing the pairwise judgment matrix and matrix mathematical method, the importance is ranked. From the perspective of psychology, too many grades will affect the difficulty of judgment, so in the evaluation process of multiple acoustic data and wind instruments, the 1 - to 9 -scale method is the most reasonable. The results of the scale method are shown in Figure 4, where data represent different types of sound data groups.

DBN is a probabilistic generative model. In the process of building a matrix, in contrast to the traditional neural network of the discriminant model, the matrix corresponding to the generative model and the design is to establish a joint distribution between the observation data and the label although it may be subject to subjective factors influence, but by training the weights between its neurons, the entire neural network can generate training data according to the maximum probability, thereby reducing the influence of its subjective factors.

In this system, the maximum eigenvalue of the evaluation matrix $A$ is obtained. After normalization, the relative importance ranking is reflected. Although this structure can reduce the interference of other factors and objectively reflect the difference of influence, in the process of identifying multiple acoustic data and wind instruments, there will inevitably be a certain degree of misclassification, which may lead to inaccurate results. If the misclassification factor of low probability is not considered, the minimum value of objective function is obtained. It shows that the fitness value is the best, and the closer to the minimum value, the better the performance. The simulation results are shown in Figure 5. The vertical axis is the fitness value, and the horizontal axis is the sound data recognition error.

If the vector $V_{i}=\left(V_{i 1}, V_{i 2}, \ldots, V_{i D}\right)^{T}$ of the $i-$ th matrix, the individual extremum is set as $P_{i}=\left(P_{i 1}, P_{i 2}, \ldots, P_{i D}\right)^{T}$ and the extremum representation of all sets is $P_{c}=\left(P_{c 1}, P_{c 2}, \ldots, P_{c D}\right)^{T}$. The calculation equation of the algorithm is as follows: 


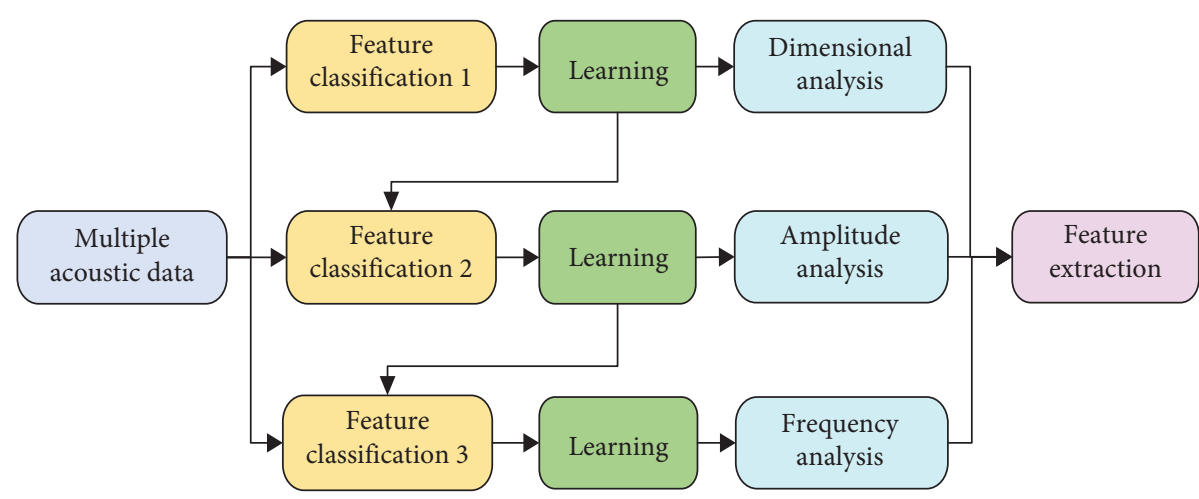

FIGURE 3: The analysis process of acoustic data from three dimensions.

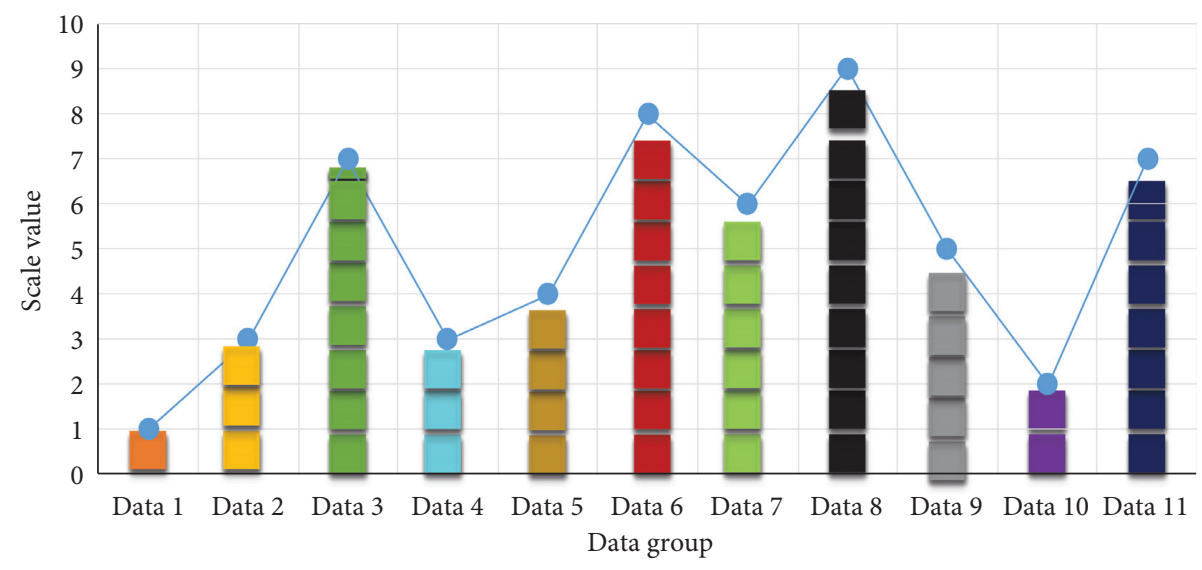

Figure 4: Simulation results of the wind instrument feature extraction model.

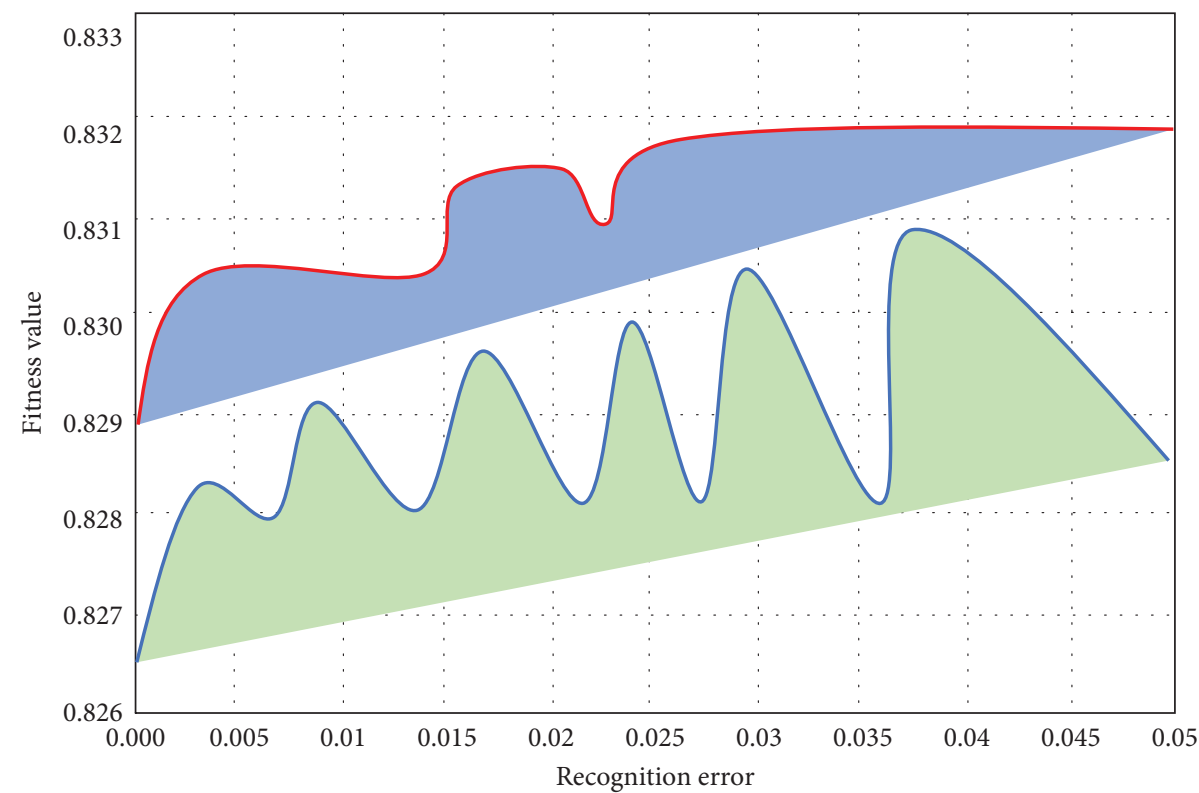

- Not optimized

- Optimized

FIGURE 5: Simulation results of the wind instrument feature extraction model. 


$$
V_{i}=\frac{\sum_{i=1}^{D} V_{i 1}}{D},
$$

where $D$ is the inertia weight, $i$ represents the current number of iterations, and $V_{i D}$ is the discrimination speed. After that, we need to use the consistency test, which is represented by $\mathrm{CI}$, and the calculation formula can be expressed as follows:

$$
\mathrm{CI}=\frac{\lambda_{\max }-n}{n-1}
$$

According to the calculated recognition similarity value to find the evaluation similarity QR, Table 1 shows the average random consistency index ES value.

The defects of the comprehensive evaluation method in this model are also very obvious. Therefore, combined with audiology and time-domain signal analysis, in the unified analysis of the detection process of multiple acoustic data, the acoustic recognition method based on three-layer encryption protocol is combined, and the essence of the deep confidence network algorithm is applied to this instrument recognition evaluation model. Based on the feature extraction factor, the rules and process of the wind instrument scoring test are determined, which are divided into data collection, data processing, result feedback, and other parts. In the application of the conventional comprehensive evaluation method, the verification is carried out gradually from the top to the bottom, and the consistency test is required in every step. The process is too cumbersome, and even if the test is reasonable, the overall consistency test is also required. Therefore, in the actual calculation, there will be the results with high consistency between the calculation results and the actual situation, and the data processing results are shown in Figure 6.

Next, the evaluation method is optimized. In the research of this paper, the method is mainly improved by using the 3scale method, which is more simple to operate. Only through the comparison between the two, the calculation is carried out according to the importance degree example. If the factors are equally important, 1 is used and 0.5 is not the same. Although the algorithm has the characteristics of simple operation, it has other defects. For example, personal preference will directly affect the establishment of the judgment matrix. It is very easy to make objective mistakes due to subjective factors and cannot pass the consistency test. Moreover, these factors are very complex, but after optimization, the algorithm can not pass the consistency test. The objectivity of the evaluation can be greatly changed. Therefore, through the simulation verification, the evaluation method after this optimization can promote the improvement of the multiacoustic data in the identification of the instrument and further improve the quality of the multiacoustic data and the identification of the instrument. Under different conditions, the evaluation results are shown in Figure 7.

In the process of evaluation, the domain of hypothesis factor is represented by $j$, the domain of evaluation grade is represented by $f_{i}\left(x_{i}\right)$, the membership degree of factor $g$ to $h$ is represented by $\lambda, w$ is the dimension of acoustic data, $C$ is a nonzero constant, and the vector $v$ of evaluation function is expressed as follows:

$$
\begin{aligned}
v_{1} & =\sum_{i=1}^{n}\left(g_{i} f_{i}\left(x_{i}\right)+\frac{1}{2} h_{i} f_{i}\left(x_{i}\right)^{2}\right)+C, \\
v_{2} & =\frac{1}{2} \lambda \sum_{j=1}^{T} w_{j}^{2}+C, \\
v & =\frac{v_{1}+v_{2}}{2} .
\end{aligned}
$$

\subsection{Evaluation and Simulation Process of Wind Instrument} Feature Extraction Evaluation System. In the process of extracting the characteristics of the instrument with multiacoustic data, it is necessary to transform the data of multiacoustic data and instrument types quantitatively. Therefore, in order to solve the weight problem of subjective evaluation in the recognition process, this study summarizes the disadvantages of traditional sound recognition technology, combines an interactive model based on the neural network optimization algorithm and deep self-study network classification algorithm, and then realizes the modeling and analysis of the recognition process by treating the output and input of the measurement data. Finally, the model can effectively reduce the scoring error in the process of different acoustic data and instrument feature recognition, improve the evaluation accuracy and objective efficiency, can be used for different multiacoustic data, the characteristics recognition, and distributed management of the instrument, and can reduce the subjective interference factors in the comprehensive evaluation model.

In addition, the acoustic data analysis model based on the neural network optimization algorithm and the deep self-learning network classification algorithm will also have lower computational complexity than the acoustic data analysis model based on the conventional optimization algorithm. This is because the neural network algorithm and the deep self-learning network are used. When the classification algorithm processes data, it will perform a certain degree of self-training, and as the number of training increases, it will reduce the subsequent computational complexity and computational cost (data cloud storage and realtime calling technology).

In the simulation of the instrument identification system based on multiacoustic data, the gray number involved is composed of real number, and its position is very important and the weight is different. In the evaluation of recognition effect, it involves various factors, which need to be judged and which factors are more important. In the application of the deep confidence network classification algorithm, the original data to be measured are transformed initially, and then the reference data column is made. The relationship or association degree of different simulation data columns is calculated, and then the correlation degree is sorted. In the process of identifying multiacoustic data and instrument, the 
TABle 1: Common criteria for evaluating similarity ES.

\begin{tabular}{cccccccccccc}
\hline$n$ & 1 & 2 & 3 & 4 & 5 & 6 & 7 & 8 & 9 & 10 & 11 \\
\hline QR & 0.04 & 0.19 & 0.26 & 0.33 & 0.57 & 0.66 & 0.77 & 0.89 & 0.93 & 0.97 & 1.00 \\
\hline
\end{tabular}

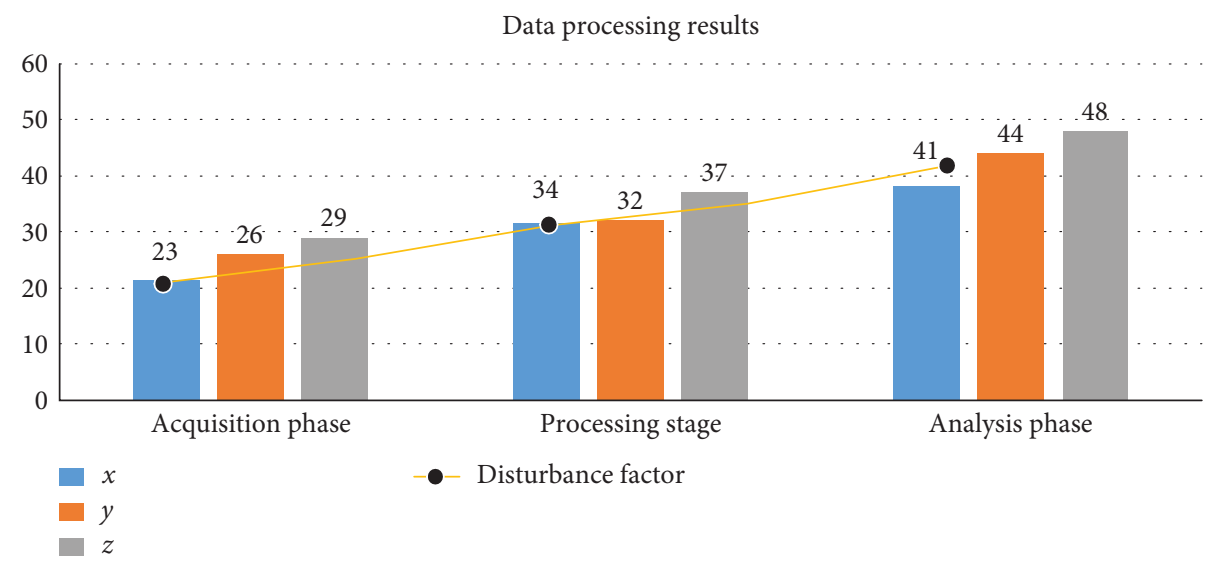

FIgURE 6: The result of data processing under this model.

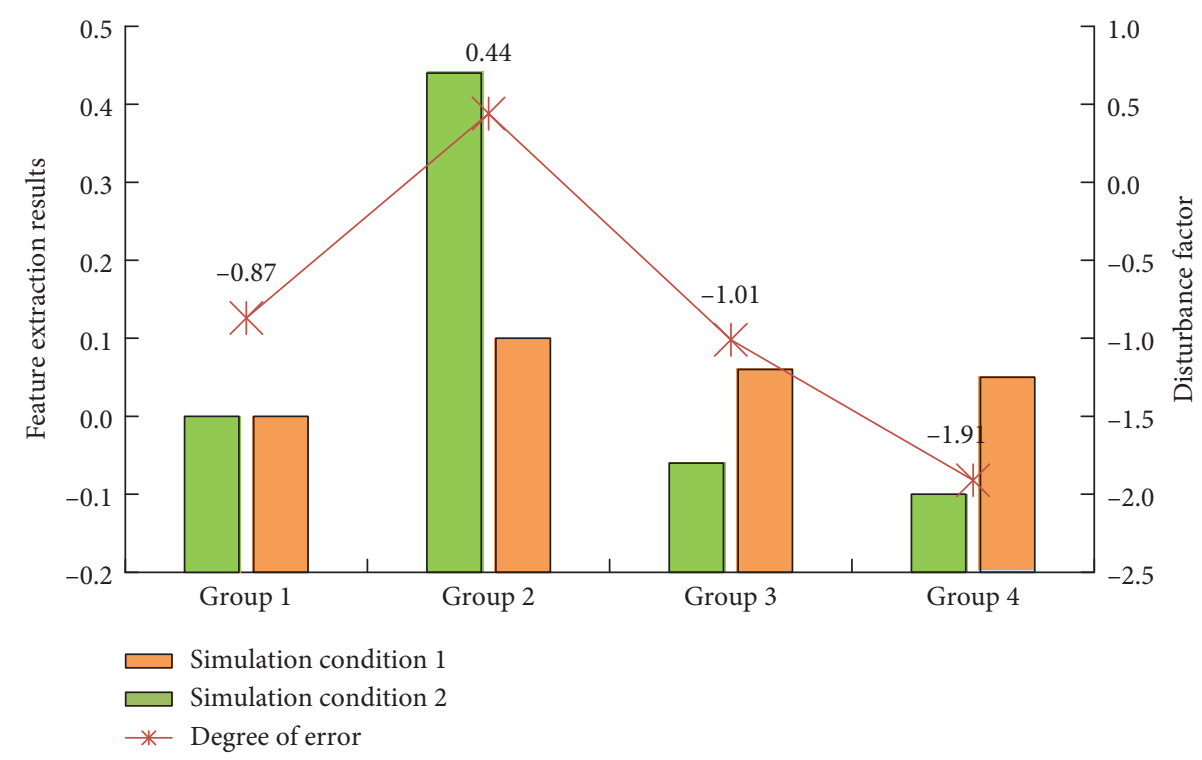

FIgURE 7: Evaluation results of the data obtained by this model.

meaning of different acoustic data is different, so it cannot be analyzed equally. The original data need to be processed by feature extraction. The feature extraction process after data analysis is shown in Figure 8.

The absolute difference of the characteristic relationship of each factor at the same observation point is calculated:

$$
\Delta t=\frac{\sum_{i=1}^{t} x_{i}}{l}
$$

where $l$ is the acoustic coincidence degree and $x_{i}$ is the $i$ - th acoustic data. Next, we need to calculate the correlation between each subfactor and the main factor in the simulation process. When making a comprehensive evaluation of things, most of the cases will involve the problem of sorting. The evaluation objects need to be sorted first, so the gray comprehensive evaluation is also needed.

$$
\eta\left(x_{i}\right)=\frac{1}{\Delta t}\left(\sum_{i=1}^{t}\left(x_{i}-x_{i-1}\right)+\frac{i}{i-1}\right)
$$

where $\eta\left(x_{i}\right)$ is the discriminant factor and $x_{i}$ is the $i-$ th acoustic data. Then, the evaluation system is established for the simulation results, and the improved algorithm proposed in this paper is used to determine the weight of each index, improve the accuracy of the index weight, and ensure that the weight distribution is more real. In this study, the evaluation level is set to 5 . 


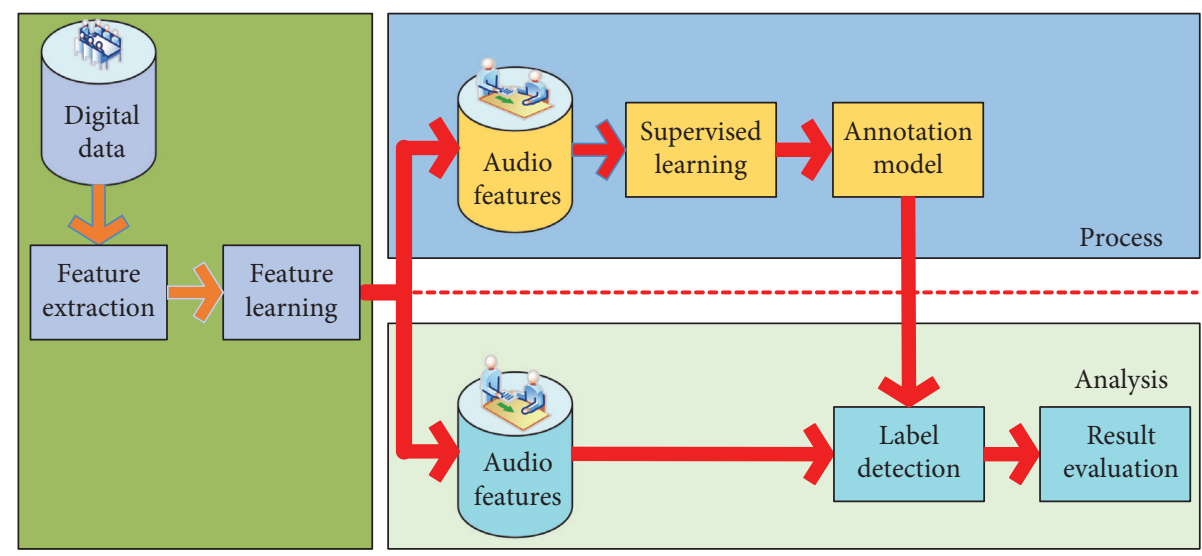

FIGURE 8: Feature extraction simulation results obtained after data analysis.

\section{Application Effect Analysis of Multiple Acoustic Data in Wind Instrument Feature Extraction}

4.1. Experimental Design Part. Before the formal experiment of the recognition model, the recognition and evaluation rules are determined according to the experimental samples, and the rules are screened for the multiple acoustic data and the characteristic parameters of wind instruments. The experimental results are shown in Figure 9. The experimental objects are classified by deep confidence network, and the $L$ times of screening and classification are carried out, and the $L$ - th time of classification results is taken as the final experimental results.

It can be seen from Figure 9 that with the increase in the key feature parameters and the number of experiments, the corresponding change trends of the two groups of experimental groups and the control group show great differences, and the coupling errors also show different regular changes, which also shows that the multiple acoustic data analysis features are essentially different after the deep belief network classification. Therefore, the multiple acoustic data analysis model is more stable and accurate than the traditional acoustic data analysis model.

In this study, there is no clear causal relationship between the research object and the results of the research index because multiple acoustic data may mean more wind instrument characteristics, and conversely, the more wind instrument characteristics may also contain more acoustic data. Therefore, in this study, the independent variable and the dependent variable of the study are not distinguished, but an objective evaluation method is used.

In this multiple acoustic data and wind instrument recognition model, this study uses five classification methods, from excellent to unqualified. After determining the grading, these grades are assigned a score. Five grades are assigned a score by 10 points, namely, excellent grade score interval $[8,10]$, good grade score interval $[6,8]$, qualified grade score interval $[4,6]$, basic qualified grade score interval $[2,4]$, and unqualified grade score interval [0, 2]. Table 2 shows the preliminary evaluation results of the experiment.
In addition, in order to evaluate the accuracy of the results of this model, the existing experts constitute a panel of judges and grade the experimental objectives according to the evaluation index scoring standard. Considering that there are 19 third-level indicators, these indicators are very large data. In order to facilitate calculation, the matrix is divided into blocks in the research and analysis of this model. The first level index is evaluated comprehensively by using the gray theory, and the top-level evaluation target is evaluated comprehensively. To facilitate calculation, the indexes are numbered first and then the weight is calculated. Table 3 shows the statistics of data results evaluated and identified by professional vocal teachers.

4.2. Data Processing and Result Analysis. Figure 10 shows the evaluation result of the wind instrument feature extraction model based on multiple acoustic data analysis. The relevant evaluation data in the wind instrument feature extraction model are processed by MATLAB software.

It can be seen from Figure 10 that with the increase in the efficiency of key feature parameter recognition, the methods using deep confidence networks and those without deep confidence networks have different regular changes, and the number of recognized features is also different. According to the evaluation results in Figure 10, the evaluation index system of the recognition system can be divided into three levels. There are four secondary indicators, which are multiple acoustic data recognition score, feature timbre score, wind instrument recognition accuracy score, and instrument recognition accuracy score. Each multiple acoustic data indicator is divided into three different levels, and the instrument recognition score has three levels. This index system can comprehensively reflect the recognition accuracy of the system for multiple acoustic data and wind instruments. In the data processing of the experimental results, a simple weighted average method is used to calculate the total score. It can be seen from the results in Figure 10 that the accuracy of this model for multiple acoustic data and wind instrument recognition is still very high. However, this data analysis model still has some shortcomings. For example, it only considers the acoustic 


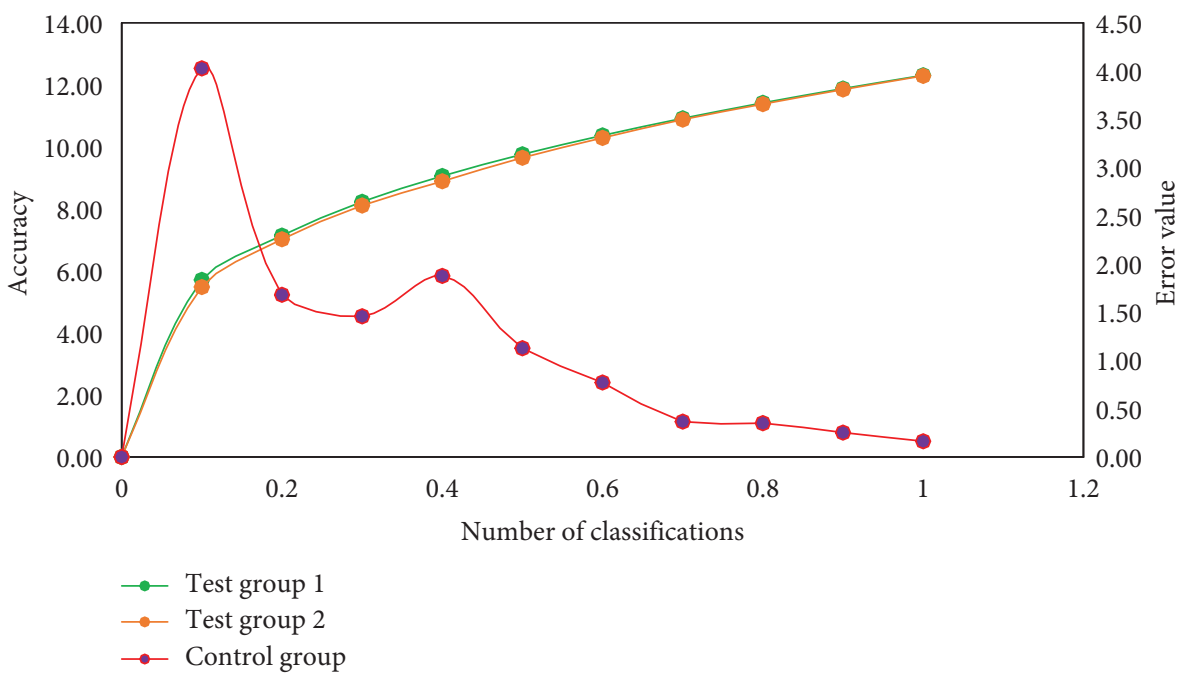

Figure 9: Preliminary results of the experiment.

Table 2: Preliminary evaluation results of experiments.

Category

Single-dimensional acoustic data

Multidimensional acoustic data

Wind instrument no. 1 Wind instrument no. 2 Wind inst

\begin{tabular}{llll}
7.2 & 7.6 & 8.3 & 8.1 \\
8.6 & 8.9 & 9.5 & 9.6 \\
\hline
\end{tabular}

TABLE 3: Evaluation and recognition of experimental targets by professional music teachers.

\begin{tabular}{lcccc}
\hline Category & Wind instrument no. 1 & Wind instrument no. 2 & Wind instrument no. 3 & Wind instrument no. 4 \\
\hline Single-dimensional acoustic data & 7.9 & 8.5 & 8.9 & 9.0 \\
Multidimensional acoustic data & 8.5 & 8.6 & 9.3 & 9.6 \\
\hline
\end{tabular}

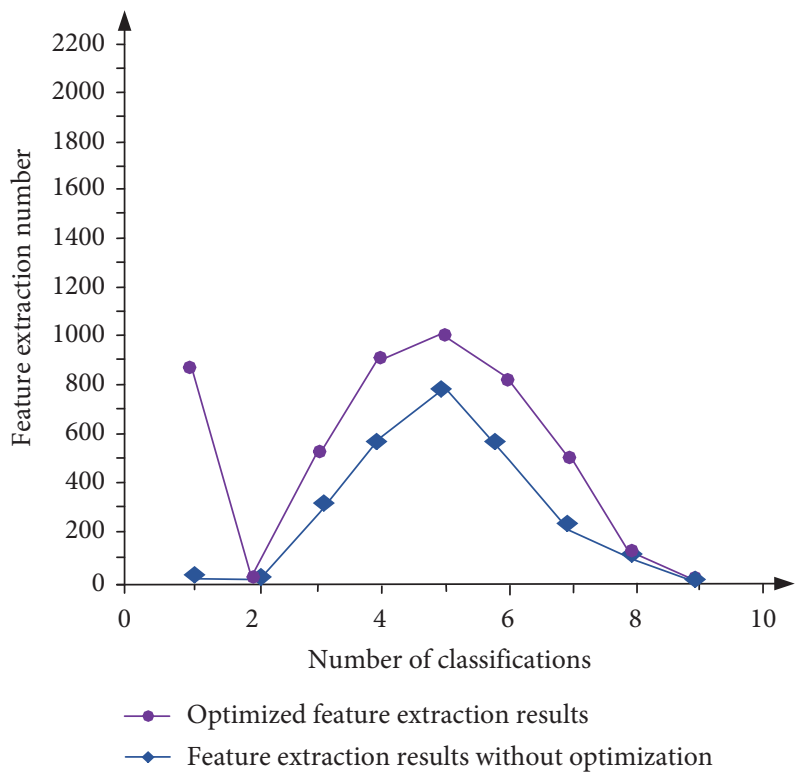

FIGURE 10: Evaluation results of the experimental model.

data types of known wind instruments and does not classify and process them according to the differences in timbre. Therefore, it may be difficult to identify aging wind instruments and unknown instruments. There is a very low probability of misjudgment.

\section{Conclusion}

The current common wind instrument feature extraction methods have problems such as a large proportion of subjective factors and low intelligence. Based on these problems, this paper studies a wind instrument feature extraction system based on a deep belief network classification algorithm and multiple acoustic data. The first point is the use of autocorrelation function and gray-scale fuzzy evaluation function. The audio signals of different multiple acoustic data are processed first, and the maximum value of the autocorrelation function curve in the pitch period is used to realize preliminary identification and then classify the deep confidence network. The error of the algorithm is analyzed. The second point is to construct the application of the deep belief network classification algorithm in the recognition of musical instruments. In the evaluation, the comprehensive evaluation method is adopted, and then the theoretical level is analyzed to improve the deficiencies of the comprehensive evaluation method. The third point is to use the deep belief network classification algorithm for comprehensive consideration to establish an adaptive identification system. In the fourth point, through design experiments, experiments show that the wind instrument feature extraction system based on the deep belief network classification algorithm and multiple acoustic data analysis has the advantages of good reliability, high intelligence, and 
strong resistance to subjective factors, which confirms the advantages of multiple acoustic data analysis. Effectiveness in feature extraction of wind instruments. However, this study only considered the processing of multiple acoustic signals and did not consider the noise elimination, so further research can be carried out.

\section{Data Availability}

The data used to support the findings of this study are available from the corresponding author upon request.

\section{Conflicts of Interest}

The authors declare that they have no conflicts of interest.

\section{Acknowledgments}

This research was supported by Art Science Planning Project of Heilongjiang Province: Research on the develop (No. 2020B052).

\section{References}

[1] M. Akhavan Aghdam, A. Sharifi, and M. M. Pedram, "Combination of rs-fMRI and sMRI data to discriminate autism spectrum disorders in young children using deep belief network," Journal of Digital Imaging, vol. 31, no. 2, pp. 1-9, 2018.

[2] G. G. Wind, S. Platnick, K. Meyer et al., “The Chimaera system for retrievals of cloud top, optical and microphysical properties from imaging sensors," Computers \& Geosciences, vol. 134, Article ID 104345, 2019.

[3] P. Priyadarshinee, "Deep learning for acoustic signal processing for industrial noise," The Journal of the Acoustical Society of America, vol. 148, no. 4, Article ID 2767, 2020.

[4] K. Leftwich, G. Drouant, and J. W. Ioup, "Determining the range to marine mammals in the Northern gulf of Mexico via bayesian acoustic signal processing," The Journal of the Acoustical Society of America, vol. 148, no. 4, Article ID 2589, 2020.

[5] H. Relao-Iborra, J. Zaar et al., "A speech-based computational auditory signal processing and perception model," The Journal of the Acoustical Society of America, vol. 146, no. 5, Article ID 3306, 2019.

[6] S. Gannot and P. A. Naylor, "Highlights from the audio and acoustic signal processing technical committee [in the spotlight]," IEEE Signal Processing Magazine, vol. 36, no. 2, pp. 136-134, 2019.

[7] M. Guo, Y. Ma, X. Yang, and R. W. Mankin, "Detection of damaged wheat kernels using an impact acoustic signal processing technique based on Gaussian modelling and an improved extreme learning machine algorithm," Biosystems Engineering, vol. 184, pp. 37-44, 2019.

[8] A. Ernoult, C. Vergez, S. Missoum, P. Guillemain, and M. Jousserand, "Woodwind instrument design optimization based on impedance characteristics with geometric constraints," The Journal of the Acoustical Society of America, vol. 148, no. 5, Article ID 2864, 2020.

[9] Q. Wang, B. Zhou, Y. Chen, and H. Quan, "Subarray beamspace adaptive beamforming combined with array shape estimation based on non-acoustic sensor," Acoustical Physics, vol. 65, no. 2, pp. 226-233, 2019.
[10] L. Pan, C. He, Y. Tian, H. Wang, X. Zhang, and Y. Jin, “A classification-based surrogate-assisted evolutionary algorithm for expensive many-objective optimization," IEEE Transactions on Evolutionary Computation, vol. 23, no. 1, pp. 74-88, 2019.

[11] W. Wang, H. Liu, J. Yang, G. Cao, and C. Hua, "Speech enhancement based on noise classification and deep neural network," Modern Physics Letters B, vol. 33, no. 17, Article ID 1950188, 2019.

[12] P. Perera and V. M. Patel, "Learning deep features for oneclass classification," IEEE Transactions on Image Processing, vol. 28, no. 11, pp. 5450-5463, 2019.

[13] G. Martin, S. Kaviraj, A. Hocking et al., "Galaxy morphological classification in deep-wide surveys via unsupervised machine learning," Monthly Notices of the Royal Astronomical Society, vol. 491, no. 1, p. 1, 2019.

[14] I. Lagoida, S. Voronov, and V. Mikhailov, "Characteristics of forbush decreases measured by the PAMELA instrument during 2006-2014," Physics of Atomic Nuclei, vol. 82, no. 6, pp. 750-753, 2019.

[15] M. Rollins, T. W. Leishman, and E. J. Hunter, "Effects of room acoustics on talkers: a use case for speech-acoustic signal processing in architectural acoustics," The Journal of the Acoustical Society of America, vol. 146, no. 4, Article ID 2794, 2019.

[16] V. Vidhyavathi, H. S. Iqbal, K. Kanthamani et al., "Performance characteristics of an instrument-free point-of-care CD4 test (VISITECT ${ }^{\circledR} \mathrm{CD} 4$ ) for use in resource-limited settings," The Journal of International Medical Research, vol. 48, no. 9, Article ID 300060520955028, 2020.

[17] B. Liu, X. Yu, A. Yu, P. Zhang, G. Wan, and R. Wang, "Deep few-shot learning for hyperspectral image classification," IEEE Transactions on Geoscience and Remote Sensing, vol. 57, no. 4, pp. 2290-2304, 2019.

[18] S. Dora, S. Sundaram, and N. Sundararajan, "An interclass margin maximization learning algorithm for evolving spiking neural network," IEEE Transactions on Cybernetics, vol. 49, no. 3, pp. 989-999, 2019.

[19] A. Gautam and V. Singh, "CLR-based deep convolutional spiking neural network with validation based stopping for time series classification," Applied Intelligence, vol. 50, no. 3, pp. 830-848, 2019.

[20] X. Yu and H. Dong, "PTL-CFS based deep convolutional neural network model for remote sensing classification," Computing, vol. 100, no. 4, 2018.

[21] R. Kamaleswaran, R. Mahajan, and A. Oguz, "A robust deep convolutional neural network for the classification of abnormal cardiac rhythm using varying length single lead electrocardiogram," Physiological Measurement, vol. 39, no. 3, 2018.

[22] Y. Xu, D. Li, Z. Wang, Q. Guo, and W. Xiang, "A deep learning method based on convolutional neural network for automatic modulation classification of wireless signals," Wireless Networks, vol. 25, no. 7, pp. 3735-3746, 2019.

[23] E. P. Mercedes et al., "Deep pyramidal residual networks for spectral-spatial hyperspectral image classification," IEEE Transactions on Geoscience and Remote Sensing, vol. 57, no. 2, pp. 740-754, 2019. 